\title{
A SNP in the 3'-UTR of HSF1 in dairy cattle affects binding of target bta-miR-484
}

\author{
Q.L. Li ${ }^{1,3}$, Z.F. Zhang ${ }^{2}$, P. Xia ${ }^{1}$, Y.J. Wang ${ }^{1}$, Z.Y. Wu ${ }^{1}$, Y.H. Jia' ${ }^{1}$ S.M. Chang ${ }^{1}$ and \\ M.X. $\mathrm{Chu}^{4}$ \\ ${ }^{1}$ College of Life Sciences, \\ Edible and Medicinal Fungi Research and Development Center, \\ Langfang Teachers University, Langfang, China \\ ${ }^{2}$ Veterinary Administration Office, Langfang Agricultural Bureau, Langfang, China \\ ${ }^{3}$ Dairy Cattle Research Center, Shandong Academy of Agricultural Science, Jinan, \\ China \\ ${ }^{4}$ Key Laboratory of Farm Animal Genetic Resources and Germplasm Innovation of \\ Ministry of Agriculture, Institute of Animal Science, Chinese Academy of Agricultural \\ Sciences, Beijing, China \\ Corresponding author: Q.L. Li \\ E-mail: liqiuling2000@126.com
}

Genet. Mol. Res. 14 (4): 12746-12755 (2015)

Received April 17, 2015

Accepted August 11, 2015

Published October 19, 2015

DOI http://dx.doi.org/10.4238/2015.October.19.18

ABSTRACT. The heat shock transcription factor 1 gene (HSF1) plays a key role in the heat stress response. We previously found a single nucleotide polymorphism (SNP) in the 3'-untranslated region (g.4693G $>\mathrm{T}$ ) of HSF1 that was related to thermo tolerance in Chinese Holstein cattle through association analysis. However, it is not known whether other SNPs also affect thermo tolerance.In this study a novel SNP, g.1451G>T, was identified by DNA sequencing and genotyped using creating restriction site-polymerase chain reaction methodology. The g.1451G $>$ T polymorphic site met Hardy-Weinberg equilibrium $(P>0.05)$. Association analysis demonstrated that this SNP had no effect on thermo tolerance traits in Holstein cattle. Findings of the study compared to the analysis of g.4693 $\mathrm{G}>\mathrm{T}$ further indicated that $\mathrm{g} .4693 \mathrm{G}>\mathrm{T}$ may play an important role in thermo tolerance, although the mechanism is not clear. RNA hybrid and 
Targetscan prediction showed that the minimum free energy hybridization of bta-miR-484 with HSF1 3'-UTR was $-31.9 \mathrm{kcal} / \mathrm{mol}$ and $\mathrm{g} .4693 \mathrm{G}>\mathrm{T}$ was in the seed sequence of bovine HSF1 that binds to bta-miR-484. Analysis by Luciferase assay indicated that HSF1 expression was directly targeted by bta-miR-484 in HEK 293T cells, and the Rluc/luc ratio of wildtype (GG) was lower than that of the mutant $(T T)(P<0.05)$. These results suggest that g.4693 G>T affects binding of HSF1 to bta-miR-484.

Key words: Dairy cattle; HSF1; bta-miR-484; Thermo tolerance; Target; Single nucleotide polymorphism

\section{INTRODUCTION}

MicroRNAs (miRNAs) are a class of small, non-coding RNAs with important roles in the regulation of gene expression through posttranscriptional degradation or translational repression through base pairing to target mRNAs (Bartel, 2009; Voinnet 2009). MicroRNAs are processed from hairpin precursors by the ribonuclease III-like enzyme dicer (Carrington and Ambros, 2003; Bartel, 2004). They silence genes through targeting cognate messenger RNAs (mRNAs) for degradation or translation repression, by binding to partial complementary cis-regulatory sites (miRNA binding sites) in target mRNAs.

A large number of miRNAs have been identified, and their diverse functions in plants and animals have led to widespread agreement over their importance (Buchan and Parker, 2007; Brodersen et al., 2008). Increasing evidence indicates that miRNAs play essential roles not only in basic physiological processes but also in stress responses (Wang et al., 2009; Wu et al., 2009). Stress-regulated genes encoding important transcription factors have been found to be targets of miRNAs (Sunkar et al., 2006; Reyes and Chua, 2007). Recently, miRNAs have been shown to play an important role in plant stress responses (Axtell and Bartel, 2005; Sunkar, 2010). JonesRhoades and Bartel (2004) observed that expression of miR395 was increased during sulfate starvation, indicating that miRNAs can be induced by environmental stress. Chiou et al. (2006) reported miR399 was up-regulated during phosphate deficiency. Tolerance to heat stress is also an important area of research.

Understanding the miRNA-mediated stress regulatory network may provide new tools for genetic improvement of heat tolerance in dairy cattle. Regulatory mechanisms linking miRNAs and environmental stress factors, however, are rarely reported. Binding of miRNA to mRNA is critical for regulating mRNA and protein expression levels. However, binding can be affected by single nucleotide polymorphisms (SNPs) at the miRNA target site, which can abolish binding, or create illegitimate binding sites. A g. 4693 G>T mutation in the 3'-untranslated region (UTR) of HSF1 found previously affected thermo tolerance traits in Chinese Holstein cattle through association analysis. The mRNA expression of different HSF1 genotype varied in the liver under heat stress conditions. The expression of the g.4693 G>T-TT genotype mRNA was significantly higher than the g.4693 G>T-GG genotype ( $\mathrm{P}<0.05$ ) (Li et al., 2011), but the mechanism is not known. In this study, we identified a novel SNP of HSF1 and studied its role in thermo tolerance of cattle, and further researched the effects of g. $4693 \mathrm{G}>\mathrm{T}$ on binding of HSF1 and a miRNA. 


\section{MATERIAL AND METHODS}

\section{Animals and DNA extraction}

Blood samples $(\mathrm{N}=930$ ) were collected by jugular venipuncture from multiparous Chinese Holstein cows on dairy farms. Genomic DNA was extracted using a previously described method (Huang et al., 2010), then stored at $-20^{\circ} \mathrm{C}$ until subsequent analysis. Rectal temperature, decrease rate of milk yield, and potassium content in erythrocytes were selected as criteria for a heat tolerant performance index and were measured according to the method previously described (Li et al., 2011). Rectal temperature was recorded at 09:00, 14:00, and 17:00, and the average value was calculated daily in August. Decrease rate of milk yield was calculated using the equation: decrease rate of milk yield $=($ milk3-milk8)/milk3x100\%, where milk3 is the calibrated milk yield during March and milk8 is the calibrated milk yield during August. For potassium content in erythrocytes determination, erythrocytes were diluted with distilled water, and $0.5 \mathrm{~mL}$ of the sample was diluted at a ratio of 1:3. The potassium concentration in the red blood cell was determined in the spectrophotometer of an atomic absorption system (TAS-990, Purkinje General, Beijing, China).

\section{Detection of SNPs and genotyping}

Primers (F: 5'-AAGCCACTAAACAAACAGCA-3'; R: 5'-AGTCTTCTCCAGCACCACA-3'; product size $897 \mathrm{bp}$; annealing temperature $55^{\circ} \mathrm{C}$ ) of bovine HSF1 (NCBI, NC_007311.3) were designed for fragment amplification by PCR. Polymorphic PCR amplified fragments were sent to Beijing Genomics Institute for sequencing. Sequence alignment was performed to screen SNPs of HSF1 using DNAStar software (DNASTAR, Inc., Madison, Wisconsin, USA); a single novel mutation g.1451 G>T was found. There was no natural endonuclease restriction site at this locus, and so creating restriction site-polymerase chain reaction (CRS-PCR) was used to genotype the polymorphic site. Primers for CRS-PCR (F: 5'-CCCATAGGGGCCAGTCTA-3'; R: 5'-CAAATGCCATCTCGTCC-3'; product size $263 \mathrm{bp}$; annealing temperature $59.9^{\circ} \mathrm{C}$ ) were designed to amplify the fragment covering the SNP for genotyping. Mutant amplified products were found to have a natural Xbal endonuclease restriction site. Four microlitre PCR products of HSF1 gene fragment were digested with $5 \mathrm{U}$ Xbal for $12 \mathrm{~h}$ at $37^{\circ} \mathrm{C}$. Then the digested products were genotyped by electrophoresis in 10\% polyacrylamide gel (39 acrylamide : 1 bisacrylamide).

\section{Bioinformatics analysis}

To identify the miRNA that may potentially regulate expression of $H S F 1$, and with one SNP present in the seed sequence of miRNA, we computational nominated miRNAs that might contribute to HSF1 regulation. Targetscan prediction program (http://www.targetscan.org/vert_50/) was used to identify miRNAs targeting bovine HSF1. Minimum free energy hybridization of miRNA with HSF1 3'-UTR was predicted using RNAhybrid software (http://bibiserv2.cebitec.uni-bielefeld. $\mathrm{de} / \mathrm{rnahybrid} /$ ).

\section{Construction of 3'-UTR of HSF1 vectors}

To construct 3'-UTR of HSF1 vectors, a specific forward primer HSF1-WT_F was designed to include an Xhol restriction site (bold, italicized), and a reverse primer HSF1-WT R incorporated 
a Notl restriction enzyme site. Primers design was carried out using the Primer Premier 5.0 software (PREMIER Biosoft, Palo Alto CA, USA) to amplify the 3'-UTR of HSF1 (Table 1). DNA products were extracted and purified using a gel/PCR extraction kit (Biomiga, San Diego, CA, USA). Wild (g.4693 G>T-GG), mut 1 (g.4693 G>T-TT) and Mut2 (4693-4699, GTCGGAC) fragments covering the seed sequence region of bta-miR-484 binding to HSF1 3'-UTR were cloned into the pMIR-RB-REPORT ${ }^{\mathrm{TM}}$ vector (RiboBio, Guangzhou, China). Cloning was performed and used to transform Trans5a cells (Invitrogen, Life Tech, Carlsbad, CA, USA) which were plated on agar containing $100 \mathrm{mg} / \mathrm{mL}$ ampicillin and incubated at $37^{\circ} \mathrm{C}$ overnight; colonies were screened by PCR for the presence of the insert using primers $\mathrm{F}$ and $\mathrm{R}$ listed previously. Positive colonies were cultured in $10 \mathrm{~mL}$ lysogeny broth medium (Fisher BioReagents, Ottawa, Ontario, Canada) containing $100 \mathrm{mg} / \mathrm{mL}$ ampicillin and incubated at $37^{\circ} \mathrm{C}$ overnight. Plasmids were isolated using a Plasmid Miniprep Kit (Biomiga, San Diego, CA, USA) according to the manufacturer instructions, and plasmid DNA quantified using a NanoDrop ND-1000. Plasmids were sequenced to ensure that variation between sequences was at the SNP locus.

Table 1. Primers sequences of HSF1.

\begin{tabular}{ll}
\hline Primers & Sequences of primers \\
\hline HSF1-WT & F: 5'-GGCGGCTCGAGAACCCCCCAAAGCCAAGGA-3' \\
& R: 5'-AATGCGGCCGGGAACAGACAGGGAGACCACACA-3' \\
HSF1-mut1 & F: 5'-GTGTCCAGTAGCCTGGTCCCTGGCTGGCGAG-3' \\
& R: 5'-CAGGGGACAGGCTACTGGACACCAGGCCTGCC-3' \\
HSF1-mut2 & F: 5'-GTGTCCAGGTCGGACGCCCCTGGCTGGCGGAG-3' \\
& R: 5'-CAGGGGACGTCCGACCTGGACACCAGGCCTGCC-3' \\
\hline
\end{tabular}

\section{Cell lines and reagents}

Cells (HEK 293T) were maintained in Dulbecco's Modification of Eagle's Medium (DMEM supplemented with $10 \%$ fetal bovine serum (v/v), $1 \%$ nonessential amino acids, $100 \mathrm{U} / \mathrm{mL}$ penicillin, and $100 \mathrm{mg} / \mathrm{mL}$ streptomycin), at $37^{\circ} \mathrm{C}$ with $5 \% \mathrm{CO}_{2}$, and were subcultured every other day. All reagents were from GIBCO (Gaithersburg, MD, USA). miRNA mimics and a non-target control were purchased from RiboBio (Guangzhou, China).

\section{Transient transfection, and luciferase reporter assay}

Cells were subcultured one day prior to transfection, and distributed into a 96-well culture tray containing $100 \mu \mathrm{L}$ DMEM complete media per well. Once cells were at $80-85 \%$ confluency, they were transfected with $100 \mathrm{ng}$ luciferase expression constructs and $50 \mathrm{nM}$ miRNA mimics or a non-target control without a miRNA insert, using Lipofectamine ${ }^{\mathrm{TM}} 2000$ (Invitrogen, Life Tech, Carlsbad, CA, USA). Approximately $48 \mathrm{~h}$ following transfection, cells were washed once with phosphate buffered saline and lyzed in Reporter Lysis Buffer (65 $\mu$ Lper well, Promega, Madison, WI, USA). Cell lysates were prepared and assayed for luciferase according to the manufacturer instructions (Promega, Madison, WI, USA). Transfection data were calculated from three repeated, independent transfections, using at least two independent preparations of DNA and plasmid clones. Activity was calculated as relative firefly luciferase activity at a $405 \mathrm{~nm}$ wavelength.

\section{Statistical analysis}

Genotypic frequencies, allelic frequencies, polymorphism information content (PIC), 
heterozygosity $(\mathrm{He})$, effective number of alleles $\left(N_{E}\right)$ and Hardy-Weinberg equilibrium were analyzed using the software program POPGENE32 (version1.31, Alberta, Canada). Pairwise linkage disequilibrum analysis was performed using SHEsis software (Shi and He, 2005; Li et al., 2009). Associations between genotypes of HSF1 and the heat tolerant index were analyzed using the least squares method by general linear model (GLM) procedure of SAS software (SAS Inc., Cary, North Carolina, USA). The effects of farm, genotype, birth season and parity were set as fixed effects, and the animal's additive genetic effect and permanent environmental effect of individual cows were included as random effects in the linear model, using the following equation:

$$
Y_{i j k l}=\mu+F_{i}+G_{j}+S_{k}+P_{l}+e_{i j k l}
$$

In the model above, $Y_{i j k l}$ is the observed value; $\mu$, the overall mean; $F_{i}, G_{j}, S_{k}, P_{i}$, the fixed effects of, respectively, farm, genotype, season and parity; and $e_{i j k l}$ is the random error.

The effect of bta-miR-484 on HSF1 expression was tested using a one-way ANOVA. Data were calculated as mean \pm standard error of the mean (SEM). A value of $P<0.05$ was regarded as significant.

\section{RESULTS}

\section{Identification of SNP 1451 G/T}

A novel SNP was discovered at position g.1451G>T, in the third intron of HSF1 (Figure 1).

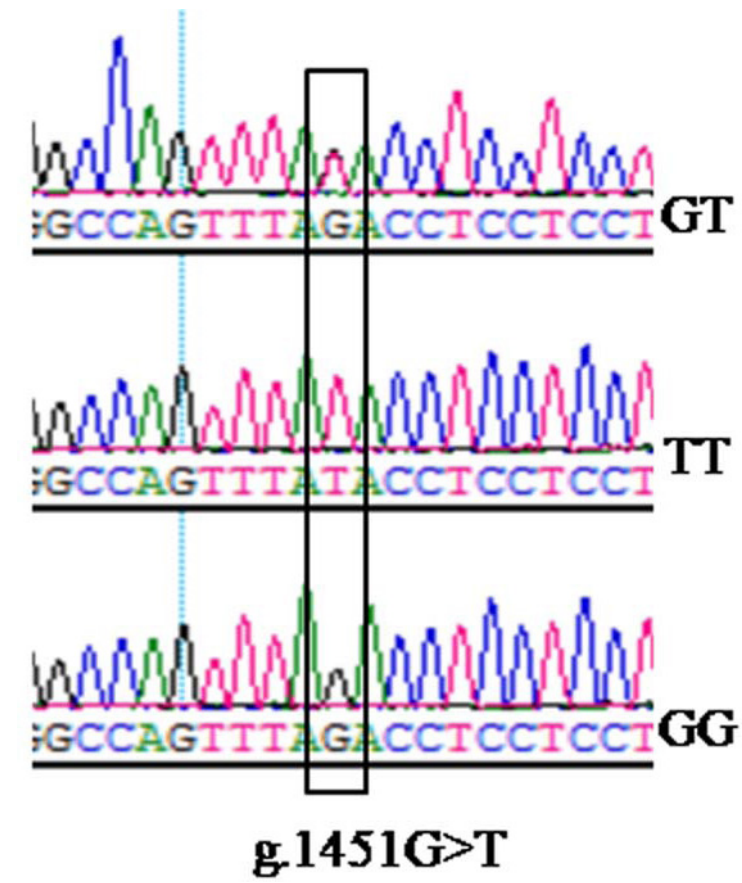

Figure 1. Sequence analysis of different genotypes of g.1451G>T of HSF1 in Chinese Holstein cattle. 
Digestion of the PCR fragment of HSF1 g.1451G $>$ T locus by Xbal produced fragments with lengths of 15 and 248 bp for genotype GG; 15, 248 and 263 bp for genotype GT; and 263 bp for genotype TT (Figure 2).

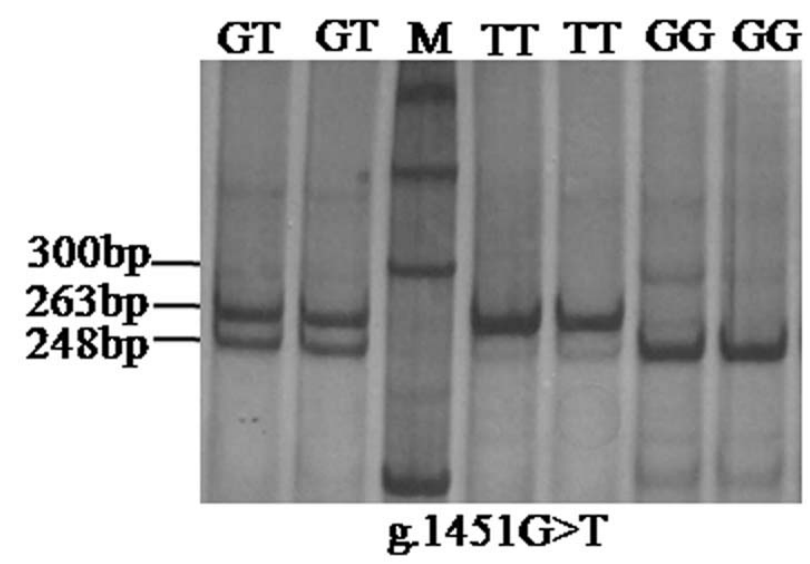

Figure 2. Genotypes of g.1451G>T of HSF1 in Chinese Holstein cattle.

At locus g.1451G>T, the genotype frequencies of GG, GT and TT were $0.6194,0.3409$ and 0.0398 , respectively. The GG genotype frequency was higher than those of genotypes GT and TT. The allelic frequency of $G$ and $T$ was 0.7898 and 0.2102 , respectively, and $G$ was the predominant allele at position g.1451G $>$ T. Results from $X^{2}$ test analysis indicated position g.1451G $>$ T met the Hardy-Weinberg equilibrium $\left(n^{2}=0.6548, P>0.05\right)$.

\section{Genetic characteristics of the locus of HSF1 in Chinese Holstein cattle}

In the Chinese Holstein population the genetic indices $H_{E}, N_{E}$, and PIC were 0.3320 , 1.4971 , and 0.2769 , respectively. The level of genetic polymorphism was moderate according to the criterion of PIC $(0.25<$ PIC < 0.5) (Vaiman et al., 1994). Pairwise linkage disequilibrium analysis showed that g.1451G $>$ T and g.4693 $\mathrm{G}>\mathrm{T}$ were not in linkage disequilibrium states $\left(D^{\prime}=\right.$ $\left.0.296, r^{2}=0.017\right)$.

\section{Association between SNP and thermo tolerance of dairy cattle}

SNP may affect phenotype,to investigate the genetic effect of SNP (g. 1451G>T), the association between SNP and thermo tolerance traits in 970 Chinese Holstein cows was analyzed. There was no significant difference in thermo tolerance traits between different genotypes (Table 2), which further confirmed that g. $4693 \mathrm{G}>\mathrm{T}$ at $3^{\prime}-\mathrm{UTR}$ of HSF1 gene, which affects thermo tolerance traits that we have previously described (Li et al., 2011), is a functional marker.

\section{Activity analysis of HSF1 3'-UTR targeted by bta-miR-484}

Targetscan predicted that bta-miR-484 is complementary to 3'-UTR of HSF1 gene. The SNP, g. $4693 \mathrm{G}>\mathrm{T}$, of HSF1 was located in the seed sequence of the binding region. The introduction of mutations disrupted base pairing between the 3'-UTR of HSF1 and bta-miR-484. 
RNAhybrid prediction showed that the minimum free energy hybridization of bta-miR-484 with HSF1 3'-UTR was $-31.9 \mathrm{kcal} / \mathrm{mol}$ (Figure 3).

\begin{tabular}{|c|c|c|c|c|}
\hline Locus & Genotype & Potassium content in erythrocytes $(\mathrm{mg} / \mathrm{L})$ & Rectal temperature $\left({ }^{\circ} \mathrm{C}\right)$ & Decrease rate of milk yield $(\%)$ \\
\hline \multirow[t]{3}{*}{ g. $1451 G>T$} & GG & $740.06 \pm 50.65$ & $39.60 \pm 0.20$ & $37.19 \pm 1.32$ \\
\hline & GT & $719.61 \pm 57.53$ & $39.56 \pm 0.24$ & $32.33 \pm 1.78$ \\
\hline & $\mathrm{TT}$ & $844.88 \pm 118.75$ & $39.09 \pm 0.78$ & $35.27 \pm 2.03$ \\
\hline
\end{tabular}

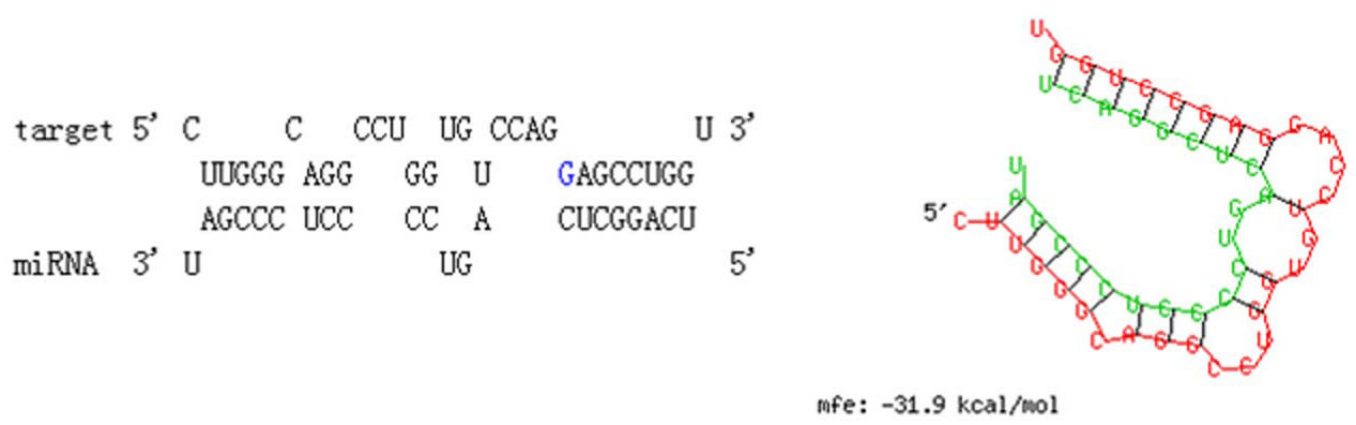

Figure 3. Interaction between bta-miR-484 and HSF1 3'-UTR. Computational modeling performed on RNAhybird software online. bta-miR-484-binding energy for the $3^{\prime}-$ UTR is shown.

To validate whether HSF1 is directly targeted by bta-miR-484 in cattle, wild HSF1 $3^{\prime}$-UTR sequence was cloned to a luciferase reporter expression vector and transfected into HEK293T cells. The repression of the luciferase activity was observed when bta-miR-484 was cotransfected with the HSF1-WT luciferase reporter vector, compared with the non-target control miRNA $(P<0.05)$ (Figure 4). Data indicated that direct binding occurred between btamiR-484 and cloned HSF1 mRNA 3'-UTR sequence, through which bta-miR-484 exerted its inhibitory effect on the upstream luciferase gene. In addition, the relative Rluc/luc ratio of bta-miR-484 cotransfected with HSF1-WT was significantly lower than that for bta-miR-484 cotransfected with HSF1-Mut1 $(P<0.05)$. There was no significant difference between btamiR-484 cotransfected with HSF1-WT and bta-miR-484 cotransfected with HSF1-Mut2 (P > 0.05) (Figure 5).

\section{Effect of SNP (g. $4693 \mathrm{G}>\mathrm{T}$ ) on targeting of bta-miR-484}

In order to investigate the binding activity of HSF1 to bta-miR-484, and whether the SNP (g. $4693 \mathrm{G}>\mathrm{T}$ ) affects this binding, three recombinant pMIR vector plasmids were successfully constructed and confirmed by sequencing. Following transient transfection of HEK293T cells, we observed that the fluorescence intensity of the T allele was higher than that of the G-allele construct at the same dose of bta-miR-484 $(P<0.05)$. The G-allele construct was silenced more efficiently than constructs with the T allele, suggesting that SNP (g. $4693 \mathrm{G}>\mathrm{T}$ ) affects the binding of HSF1 to its target bta-miR-484. 


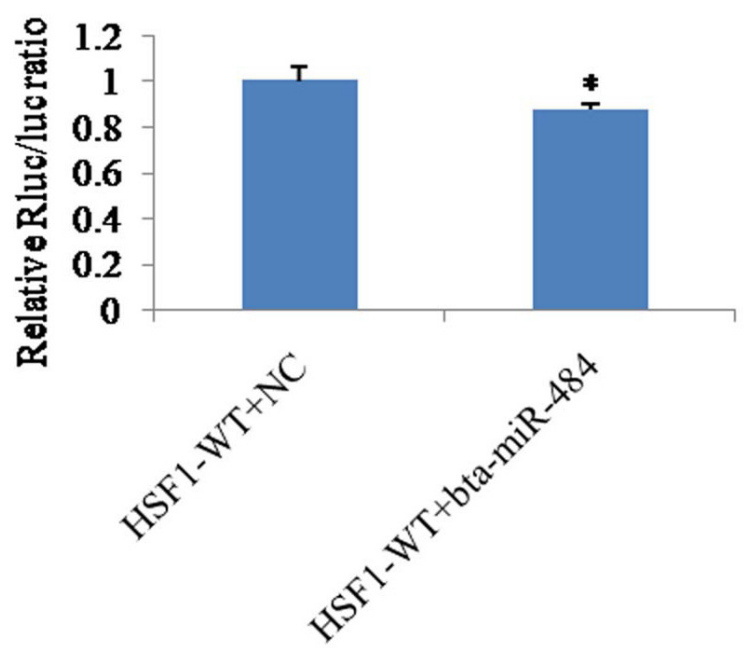

Figure 4. Bta-miR-484 decreased luciferase activity of HSF1-WT HEK 293T cells. Cells were transiently cotransfected with constructs and bta-miR-484 or scrambled with the negative control (miR-control) for $24 \mathrm{~h}$. Firefly luciferase activity was normalized to Renilla luciferase activity. Data are from three transfection experiments with assays replicated six times. WT, g. 4693 G>T-GG. Vertical bars represent SEM of six replicates.
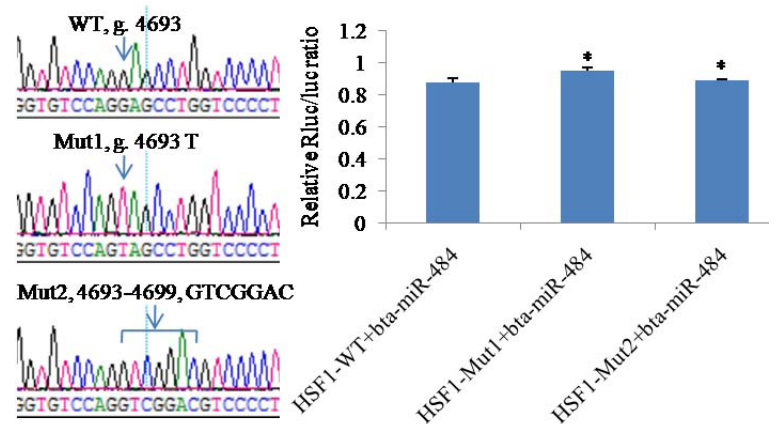

Figure 5. Cotransfection of bta-miR-484 in HEK 293T cells. Cells were transiently cotransfected with HSF1-WT, HSF1-Mut1 and HSF1-Mut2 constructs and bta-miR-484 for $24 \mathrm{~h}$. Firefly luciferase activity was normalized to Renilla luciferase activity. Data are from three replicates. WT, g. 4693 G>T-GG. Mut1, g. 4693 G>T-TT. Mut2, 4693-4699, GTCGGAC. Vertical bars represent SEM.

\section{DISCUSSION}

While stochastic or environmental effects modulate phenotypes, subjects with functional SNPs often exhibit phenotypic variation (Drogemuller et al., 2011). Heat tolerance is characterized by decreases inmilk yield, potassium content in erythrocytes, and rectal temperature (Wang et al., 2013; Fang et al., 2014). In this study, a novel SNP, g.1451G > T, was identified in the third intron of HSF1 in Chinese Holstein cattle, but was not found to affect thermo tolerance. On the other hand, another SNP that we have previously described, g. $4693 \mathrm{G}>\mathrm{T}$, located in the 3'-UTR of HSF1, was further characterized in this study and found to influence thermo tolerance. Further studies are warranted to further analyze the function of this SNP. 
Abelson et al. (2005) showed that a 3'-UTR SNP in the human gene Slit and Trk-like 1(SLITRK1) strengthens an existing miR-189 target site, thereby enhancing down regulation of SLITRK1. Another study demonstrated that a 3'-UTR SNP in sheep myostatin gene (Gdf8) creates a new, illegitimate miRNA target site, leading to significantly decreased expression of Gdf8, and contributing to the development of muscular hypertrophy (Clop et al., 2006). Micro RNAs regulate a variety of biological processes, such as cell growth, differentiation and apoptosis (Chen et al., 2004; Esau et al., 2004; Fazi et al., 2005; Chen et al., 2006; Krichevsky et al., 2006). From this, it is apparent that genetic polymorphisms residing in miRNA-binding sites can alter miRNA-target interactions, resulting in differencesin target gene expression, which could in turn influence individual performances. In this work, we found that bta-miR-484 directly bound to the 3'-UTR of HSF1. It has been previously shown, by northern blot analysis, that $m i R-484$ is predominantly located in the cytoplasm (Wang et al., 2014). We compared the sequence of bta-miR-484 with that of 3'UTR of HSF1 and observed that the latter contains a target site for bta-miR-484.

Micro RNAs regulate gene expression by binding to the 3'-UTR of target gene transcripts (Bartel, 2009). Variants, such as SNPs in miRNA regulatory regions, may result in altered mRNA or protein levels, and subsequent disease. The seed region of the miRNA (nucleotides 2-7 of the 5'end) is considered one of the most important regions determining mRNA-targeting efficacy (Bartel, 2009). In particular, miRNAs require almost perfect complementarities at the seed sites to bind targets (Brennecke et al., 2005). In the present study, the SNP g. 4693 G>T was found to occur in the seed region of the bta-miR-484 binding site; the complementary nature of these sequences led us to consider whether bta-miR-484 could directly interact with HSF1.

A wild type luciferase construct of the 3'-UTR of HSF1 (Luc- HSF1-WT), and two mutated forms (Luc-HSF1-mut1 and Luc-HSF1-mut2) were produced, and bta-miR-484 was found to suppress the luciferase activity of HSF1, but had less effect on the mutated forms than on the wild type. The introduction of mutations disrupted base pairing between the HSF1 3'-UTR and bta-miR-484, indicating that the recognition of bta-miR-484 to HSF1 occurs in a sequence-specific manner. These results suggest that HSF1 interacts with bta-miR-484 via this putative binding site. We also observed that g. $4693 \mathrm{G}>\mathrm{T}$, located in the 3'-UTR of HSF1, alleviated binding between HSF1 and bta-miR-484; this result was verified by in vitro transfection experiments.

Growing evidence indicates that complex crosstalk between environmental stressors and miRNAs exists, but regulatory mechanisms remain unclear. From this study, we conclude that altered bta-miR-484 binding to the 3'-UTR of HSF1 may participate in regulation of HSF1 expression, and may be involved in the heat stress response in dairy cattle.

\section{Conflicts of interest}

The authors declare no conflict of interest.

\section{ACKNOWLEDGMENTS}

Research supported by National Natural Science Funds (\#31402056, \#31340067); Youth Talent Project in Hebei Province (\#BJ2014048); Agricultural Science and Technology Innovation Program (\#ASTIP-IAS13); and the Doctoral Foundation of Langfang Teachers University (\#LSLB201404), in China. 


\section{REFERENCES}

Abelson JF, Kwan KY, O'Roak BJ, Baek DY, et al. (2005). Sequence variants in SLITRK1 are associated with Tourette's syndrome. Science 310: 317-20.

Axtell MJ and Bartel DP (2005). Antiquity of microRNAs and their targets in land plants. Plant Cell 17: 1658-73.

Bartel DP (2004). MicroRNAs: genomics, biogenesis, mechanism, and function. Cell 116: 281-97.

Bartel DP (2009). MicroRNAs: target recognition and regulatory functions. Cell 136: 215-33.

Brennecke J, Stark A, Russell RB and Cohen SM (2005). Principles of microRNA-target recognition. PLoS Biol. 3: e85.

Brodersen P, Sakvarelidze-Achard L, Bruun-Rasmussen M, Dunoyer P, et al. (2008). Widespread translational inhibition by plant miRNAs and siRNAs. Science 320: 1185-90.

Buchan JR and Parker R (2007). Molecular biology. The two faces of miRNA. Science 318: 1877-8.

Carrington JC and Ambros V (2003). Role of microRNAs in plant and animal development. Science 301: 336-8.

Chen CZ, Li L, Lodish HF and Bartel DP (2004). MicroRNAs modulate hematopoietic lineage differentiation. Science 303: 83-6.

Chen JF, Mandel EM, Thomson JM, Wu Q, et al. (2006). The role of microRNA-1 and microRNA-133 in skeletal muscle proliferation and differentiation. Nat. Genet. 38: 228-33.

Chiou TJ, Aung K, Lin SI, Wu CC, et al. (2006). Regulation of phosphate homeostasis by MicroRNA in Arabidopsis. Plant Cell 18: 412-21.

Clop A, Marcq F, Takeda H, Pirottin D, et al. (2006). A mutation creating a potential illegitimate microRNA target site in the myostatin gene affects muscularity in sheep. Nat. Genet. 38: 813-8.

Drogemuller C, Reichart U, Seuberlich T, Oevermann A, et al. (2011). An unusual splice defect in the mitofusin 2 gene (MFN2) is associated with degenerative axonopathy in Tyrolean Grey cattle. PLoS One 6: e18931.

Esau C, Kang X, Peralta E, Hanson E, et al. (2004). MicroRNA-143 regulates adipocyte differentiation. J. Biol. Chem. 279: 52361-5.

Fang W, He J, Huang J, Ju Z, et al. (2014). Study on genetic variations of PPARalpha gene and its effects on thermal tolerance in Chinese Holstein. Mol. Biol. Rep. 41: 1273-8.

Fazi F, Rosa A, Fatica A, Gelmetti V, et al. (2005). A minicircuitry comprised of microRNA-223 and transcription factors NFI-A and C/EBPalpha regulates human granulopoiesis. Cell 123: 819-31.

Huang J, Wang H, Wang C, Li J, et al. (2010). Single nucleotide polymorphisms, haplotypes and combined genotypes of lactoferrin gene and their associations with mastitis in Chinese Holstein cattle. Mol. Biol. Rep. 37: 477-83.

Jones-Rhoades MW and Bartel DP (2004). Computational identification of plant microRNAs and their targets, including a stress-induced miRNA. Mol. Cell 14: 787-99.

Krichevsky AM, Sonntag KC, Isacson O and Kosik KS (2006). Specific microRNAs modulate embryonic stem cell-derived neurogenesis. Stem Cells 24: 857-64.

Li QL, Ju ZH, Huang JM, Li JB, et al. (2011). Two novel SNPs in HSF1 gene are associated with thermal tolerance traits in Chinese Holstein cattle. DNA Cell. Biol. 30: 247-54.

Li Z, Zhang Z, He Z, Tang W, et al. (2009). Apartition-ligation-combination-subdivision EM algorithm for haplotype inference with multiallelic markers: update of the SHEsis (http://analysis.bio-x.cn). Cell Res. 19: 519-23.

Reyes JL and Chua NH (2007). ABA induction of miR159 controls transcript levels of two MYB factors during Arabidopsis seed germination. Plant J. 49: 592-606.

Shi YY and He L (2005). SHEsis, a powerful software platform for analyses of linkage disequilibrium, haplotype construction, and genetic association at polymorphism loci. Cell Res. 15: 97-8.

Sunkar R (2010). MicroRNAs with macro-effects on plant stress responses. Semin. Cell. Dev. Biol. 21: 805-11.

Sunkar R, Kapoor A and Zhu JK (2006). Posttranscriptional induction of two Cu/Zn superoxide dismutase genes in Arabidopsis is mediated by downregulation of miR398 and important for oxidative stress tolerance. Plant Cell 18: 2051-65.

Vaiman D, Mercier D, Moazami-Goudarzi K, Eggen A, et al. (1994). A set of 99 cattle microsatellites: characterization, synteny mapping, and polymorphism. Mamm. Genome 5: 288-97.

Voinnet O (2009). Origin, biogenesis, and activity of plant microRNAs. Cell 136: 669-87.

Wang JW, Czech B and Weigel D (2009). miR156-regulated SPL transcription factors define an endogenous flowering pathway in Arabidopsis thaliana. Cell 138: 738-49.

Wang K, Sun T, Li N, Wang Y, et al. (2014). MDRL IncRNA regulates the processing of miR-484 primary transcript by targeting miR-361. PLoS Genet. 10: e1004467.

Wang Y, Huang J, Xia P, He J, et al. (2013).Genetic variations of HSBP1 gene and its effect on thermal performance traits in Chinese Holstein cattle. Mol. Biol. Rep. 40: 3877-82.

Wu G, Park MY, Conway SR, Wang JW, et al. (2009). The sequential action of miR156 and miR172 regulates developmental timing in Arabidopsis. Cell 138: 750-9. 